\title{
Resveratrol Enhances Inhibition Effects of Cisplatin on Cell Migration and Invasion and Tumor Growth in Breast Cancer MDA-MB-231 Cell Models In Vivo and In Vitro
}

\author{
Meng-Die Yang ${ }^{1,+}$, Yang Sun ${ }^{1,2,+}$, Wen-Jun Zhou ${ }^{1}$, Xiao-Zheng Xie ${ }^{1}$, Qian-Mei Zhou ${ }^{1}$, Yi-Yu Lu ${ }^{1}$ \\ and Shi-Bing $\mathrm{Su}^{1, *}$ \\ 1 Research Center for Traditional Chinese Medicine Complexity System, Institute of Interdisciplinary \\ Integrative Medicine Research, Shanghai University of Traditional Chinese Medicine, \\ Shanghai 201203, China; yangmengdie_yzu@foxmail.com (M.-D.Y.); bssunyang@126.com (Y.S.); \\ be6835@126.com (W.-J.Z.); YearMonthDaySH@163.com (X.-Z.X.); tazhou@163.com (Q.-M.Z.); \\ ava0048@163.com (Y.-Y.L.) \\ 2 WEIHAI WEGO BioTech CO., LTD., Weihai 264200, China \\ * Correspondence: shibingsu18@shutcm.edu.cn or shibingsu07@163.com \\ + Co-first authors.
}

check for

updates

Citation: Yang, M.-D.; Sun, Y.; Zhou, W.-J.; Xie, X.-Z.; Zhou, Q.-M.; Lu, Y.-Y.; Su, S.-B. Resveratrol Enhances Inhibition Effects of Cisplatin on Cell Migration and Invasion and Tumor Growth in Breast Cancer MDA-MB-231 Cell Models In Vivo and In Vitro. Molecules 2021, 26, 2204. https://doi.org/10.3390/ molecules26082204

Academic Editors: Tze-chen Hsieh and Joseph M. Wu

Received: 5 February 2021

Accepted: 19 March 2021

Published: 12 April 2021

Publisher's Note: MDPI stays neutral with regard to jurisdictional claims in published maps and institutional affiliations.

Copyright: (c) 2021 by the authors. Licensee MDPI, Basel, Switzerland. This article is an open access article distributed under the terms and conditions of the Creative Commons Attribution (CC BY) license (https:/ / creativecommons.org/licenses/by/ $4.0 /)$.
Abstract: Triple-negative breast cancer (TNBC) is a refractory type of breast cancer that does not yet have clinically effective drugs. The aim of this study is to investigate the synergistic effects and mechanisms of resveratrol combined with cisplatin on human breast cancer MDA-MB-231 (MDA231) cell viability, migration, and invasion in vivo and in vitro. In vitro, MTS assays showed that resveratrol combined with cisplatin inhibits cell viability as a concentration-dependent manner, and produced synergistic effects $(\mathrm{CI}<1)$. Transwell assay showed that the combined treatment inhibits TGF- $\beta 1$-induced cell migration and invasion. Immunofluorescence assays confirmed that resveratrol upregulated E-cadherin expression and downregulated vimentin expression. Western blot assay demonstrated that resveratrol combined with cisplatin significantly reduced the expression of fibronectin, vimentin, P-AKT, P-PI3K, P-JNK, P-ERK, Sma2, and Smad3 induced by TGF- $\beta 1$ $(p<0.05)$, and increased the expression of E-cadherin $(p<0.05)$, respectively. In vivo, resveratrol enhanced tumor growth inhibition and reduced body weight loss and kidney function impairment by cisplatin in MDA231 xenografts, and significantly reduced the expressions of P-AKT, P-PI3K, Smad2, Smad3, P-JNK, P-ERK, and NF- $\kappa B$ in tumor tissues $(p<0.05)$. These results indicated that resveratrol combined with cisplatin inhibits the viability of breast cancer MDA231 cells synergistically, and inhibits MDA231 cells invasion and migration through Epithelial-mesenchymal transition (EMT) approach, and resveratrol enhanced anti-tumor effect and reduced side of cisplatin in MDA231 xenografts. The mechanism may be involved in the regulations of PI3K/AKT, JNK, ERK and NF$\kappa \mathrm{B}$ expressions.

Keywords: resveratrol; cisplatin; breast cancer; MDA-MB-231 cells; epithelial-mesenchymal transition

\section{Introduction}

Breast cancer is the most common malignant tumor in women, and its incidence is on the rise [1,2]. It has become a major threat to women's health. Triple-negative breast cancer (TNBC) is the most common and invasive breast cancer subtype in younger patients and is characterized by a lack of estrogen receptor, progesterone receptor, and human epidermal growth factor receptor 2 [3]. The lack of these receptors makes TNBC aggressive and does not respond to hormones and targeted therapies. In addition, TNBC is highly metastatic and can recur within three years [4].

In the past five years, the focus of TNBC treatment research has gradually focused on molecularly targeted drugs, including epidermal growth factor receptor antibodies, small molecule single targets, and multi-target tyrosine kinase inhibitors, anti-angiogenic, 
and DNA repair drugs [5]. Advances in research are expected to provide more treatment options for TNBC patients to improve cure rates and improve prognosis [6]. Although significant advances have been made in a variety of new drugs for HER2 or ER in recent years, the progression of TNBC is limited. Compared with other subtypes of breast cancer, TNBC patients have a survival rate of only 77\% [7].

Resveratrol (trans-3,4,5-trihydroxystilbene) is a non-flavonoid polyphenol, derived from natural medicines such as Polygonum cuspidatum, Rheum palmatum, and fruits such as grapes, blueberries, mulberries and peanuts. It has been reported that resveratrol has the effect of anti-cancer, and can inhibit the occurrence and metastasis of breast cancer [8]. Previous studies showed that the anti-breast cancer effects of resveratrol includes inhibiting cell growth and proliferation by inducing autophagy [9] and apoptosis [10], reversing epithelial-mesenchymal transition (EMT) and decreasing metastasis [11,12], regulating the phase I and phase II detoxification system [13], affecting the epigenetic mechanism [14], increasing the sensitivity [11], and reducing cytotoxicity [15] of chemotherapy, suppressing multidrug resistance [16], and modulating immune response [17].

Cisplatin as a first-line drug for metastatic disease has a response rate of more than $40 \%$ in breast cancer metastasis [18]. It is a DNA-damaging drug, especially in the TNBC. Compared with other types of breast cancer, TNBC has a higher risk of distant recurrence and death in the first five years [19]. Due to the heterogeneity of TNBC, the lack of clear molecular targets [20] and the inherent genomic instability caused by TNBC lack of DNA repair may result in the production of platinum drugs (such as cisplatin or carboplatin) in the treatment of TNBC. In clinic, the addition of platinum drugs can significantly improve the pathological complete response rate in the neoadjuvant therapy of TNBC. However, cisplatin, one of the most active cytotoxic drugs at present, has a therapeutic effect on a variety of malignant tumors [21], and also produces serious side effects such as severe toxicity including nephrotoxicity [22], neurotoxicity [23], gastrointestinal toxicity [24], peripheral neuropathy [25], ototoxicity [26], and hematological toxicity [27]. Therefore, it is particularly necessary to find drugs that can reduce the side effects of cisplatin and enhance the therapeutic effects.

In this study, we investigated the synergistic effects of resveratrol combined with cisplatin on a TNBC models, MDA-MB-231 (MDA231) cell viability, migration and invasion in vivo and in vitro, and their effective mechanisms were also explored through EMT approach and the regulations of PI3K/AKT, JNK, ERK, and NF-KB signaling pathways.

\section{Results}

\subsection{Resveratrol Combined with Cisplatin Inhibits Synergistically the Activity of MDA231 Cells}

The effects of resveratrol combined with Cisplatin on the viability of MDA-MB-231 cells were detected by MTS assay. After treated with 2-64 $\mu \mathrm{M}$ Cisplatin (Figure 1A) and $12.5-250 \mu \mathrm{M}$ resveratrol (Figure 1B) for 24,48 , and $72 \mathrm{~h}$, cell viability decreased significantly $(p<0.05$ or $p<0.01)$, and was positively correlated with time and concentration, showed a dose- and time-dependent manner. The IC50 were $185 \mu \mathrm{M}$ in resveratrol combined with $14 \mu \mathrm{M}$ in cisplatin. Furthermore, MDA231 cells were treated by $14 \mu \mathrm{M}$ cisplatin was combined with resveratrol 50,100, 150, 200, and $250 \mu \mathrm{M}$ for $24 \mathrm{~h}$, the survival rates of cells were $80.7 \%, 62.4 \%, 41.9 \%, 23.3 \%$, and $10.4 \%$, respectively, compared to those of resveratrol alone, the cell survival rate decreased by $16.7 \%, 25.1 \%, 19.0 \%, 21.8 \%$, and $17.7 \%$ (Figure 1C). The CIs were $14 \mu \mathrm{M}$ cisplatin combined with $200 \mu \mathrm{M}$ resveratrol (Figure 1D). When MDA231 cells were treated by $185 \mu \mathrm{M}$ resveratrol combined with cisplatin at 4, 8, 16,32 , and $64 \mu \mathrm{M}$ for $24 \mathrm{~h}$, the survival rates of cells were $80.2 \%, 61.6 \%, 24.2 \%, 17.3 \%$, and $9.1 \%$, respectively, compared to resveratrol alone (Figure $1 \mathrm{E}$ ). The CIs were $175 \mu \mathrm{M}$ resveratrol combined with 16 or $32 \mu \mathrm{M}$ cisplatin. These results indicated that cisplatin was sensitized by resveratrol, high-dose resveratrol can enhance the efficacy of cisplatin in inhibiting tumor cell growth at low doses, and there are a synergistic effect of cisplatin and resveratrol on the viability of MDA231 cells. 
A

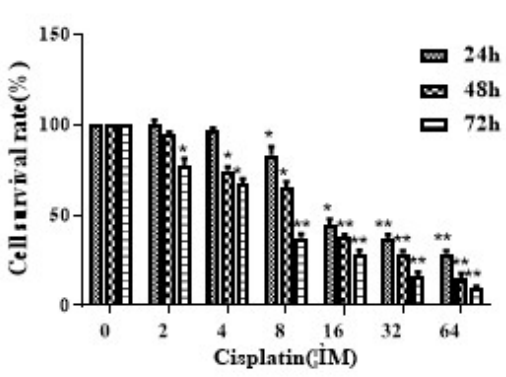

C

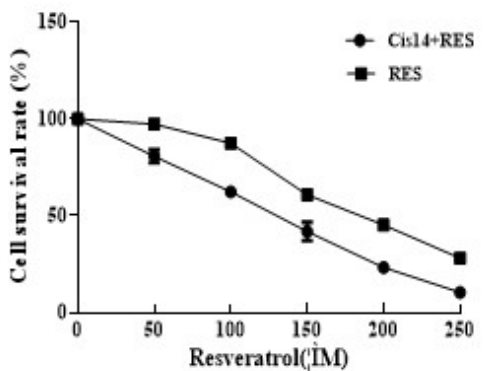

E

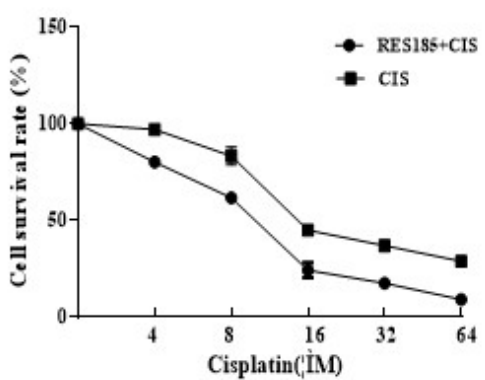

B
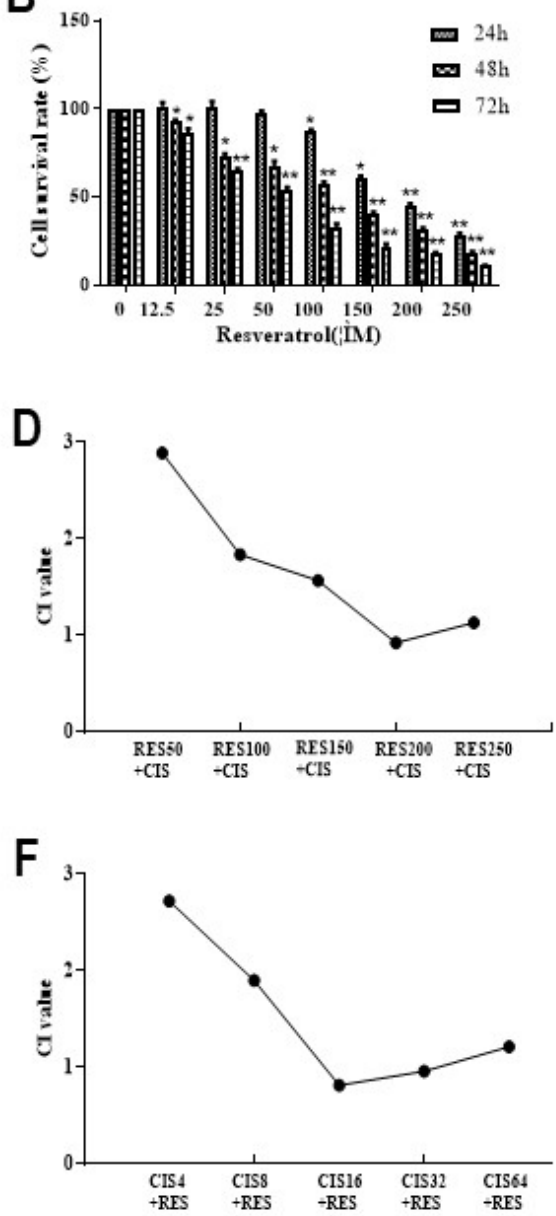

Figure 1. Effect of cisplatin and resveratrol on the viability of MDA231 cells. (A) Cell survival rate treated by cisplatin; (B) cell survival rate treated by resveratrol for 24, 48, and $72 \mathrm{~h}$; (C) cell survival rate treated by $14 \mu \mathrm{M}$ cisplatin combined with different concentrations of resveratrol for $24 \mathrm{~h}$; (D) CI value of cisplatin $14 \mu \mathrm{M}$ combined with resveratrol treatment; (E) cell survival rate treated by $185 \mu \mathrm{M}$ resveratrol combined with different concentrations of cisplatin for $24 \mathrm{~h}$; (F) CI value of $185 \mu \mathrm{M}$ resveratrol combined with cisplatin treatment. The experiment was repeated three times, RES, resveratrol. CIS, cisplatin. ${ }^{*} p<0.05,{ }^{* *} p<0.01$, vs. the control group.

\subsection{Resveratrol Combined with Cisplatin Inhibits the Migration and Invasion of MDA231 Cells}

The effects of resveratrol combined with cisplatin on the migration and invasion of MDA231 cells were detected by Transwell assay. As shown in Figure 2A,B, 12.5, $25 \mu \mathrm{M}$ and $50 \mu \mathrm{M}$ resveratrol combined with $4 \mu \mathrm{M}$ cisplatin significantly inhibited the migration of MDA231 cells compared to control group or cisplatin group $(p<0.05$ or $p<0.01)$, and the cell migration rate were $63.7 \%, 48.6 \%$, and $28.3 \%$, respectively. As shown in Figure $2 C, D$, $12.5,25,50 \mu \mathrm{M}$ resveratrol combined with $4 \mu \mathrm{M}$ cisplatin inhibited significantly the invasion of MDA231 cells, and the cell invasion rates were $66.5 \%, 61.3 \%$, and $42.8 \%$, respectively. The results showed that resveratrol combined with cisplatin can inhibit the migration and invasion of MDA231 cells. 

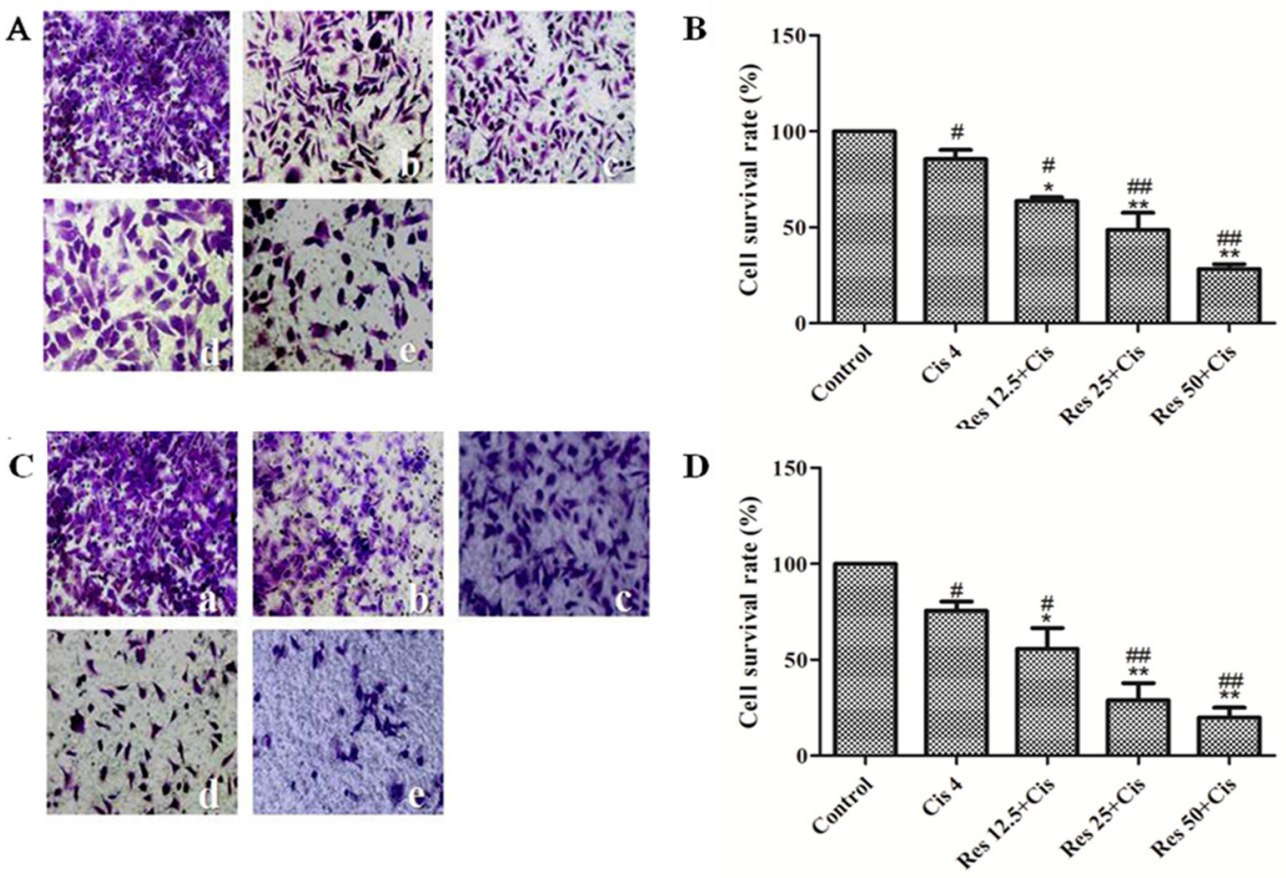

Figure 2. Effects of resveratrol combined with cisplatin on the migration and invasion of MDA231 cells. Transwell chamber migration and invasion experiments $(\times 200)$. (A) Transwell chambers images of cell migration; (B) cell migration rate; (C) transwell chambers images of cell invasion; (D) cell invasion rate. (a) Control group; (b) $4 \mu \mathrm{M}$ cisplatin group; (c) $12.5 \mu \mathrm{M}$ resveratrol $+4 \mu \mathrm{M}$ cisplatin group; (d) $25 \mu \mathrm{M}$ resveratrol $+4 \mu \mathrm{M}$ cisplatin group; (e) $50 \mu \mathrm{M}$ resveratrol $+4 \mu \mathrm{M}$ cisplatin group. \# vs. control group; ${ }^{*}$ vs. cisplatin group. ${ }^{*} / \# p<0.05,{ }^{* *} / \# \# p<0.01$, vs. the control group. Error bars represent three independent experiments, each performed three times.

\subsection{Effect of Resveratrol Combined with Cisplatin on TGF-B1-Induced Epithelial and Mesenchymal Molecular Markers in MDA231 Cells}

In order to demonstrate the effect of resveratrol combined with cisplatin on the migration and invasion of MDA231 cells whether through EMT approach, TGF- $\beta 1$-induced the changes of epithelial and mesenchymal molecular markers expressions by western blot and immunofluorescence assays. As shown in Figure 3A, the expressions of E-cadherin significantly decreased, while the expressions of vimentin and fibronectin increased by TGF- $\beta 1$ induction $(5 \mathrm{ng} / \mathrm{mL})$ in MDA231 cells, compared to control group $(p<0.01)$. Moreover, TGF- $\beta 1$ induced the expressions of E-cadherin significantly increased, while the expressions of vimentin and fibronectin decreased by resveratrol $(12.5 \mu \mathrm{M}, 25 \mu \mathrm{M}$, $50 \mu \mathrm{M})$, cisplatin $(4 \mu \mathrm{M})$, and resveratrol combined with cisplatin treatments in MDA231 cells, compared to TGF- $\beta 1$-treated group $(p<0.05)$.

Furthermore, the expression of EMT markers were verified by immunofluorescence. As shown in Figure 3C, $5 \mathrm{ng} / \mathrm{mL}$ TGF- $\beta 1$ reduced the expression of E-cadherin and increased the expression of vimentin. When $50 \mu \mathrm{M}$ resveratrol was combined with TGF- $\beta 1$, the epithelial marker E-cadherin was increased and the mesenchymal marker vimentin was decreased. There was no significant change in the expression of E-cadherin and vimentin in the combination of $4 \mu \mathrm{M}$ cisplatin and TGF- $\beta 1$. The expression of E-cadherin was increased in the combination of resveratrol, cisplatin, and TGF- $\beta 1$, and the expression of vimentin was decreased, which was consistent with the control group. These results indicated that the effects of resveratrol combined with cisplatin on the migration and invasion of MDA231 cells induced by TGF- $\beta 1$ may be involved in the regulation of EMT. 


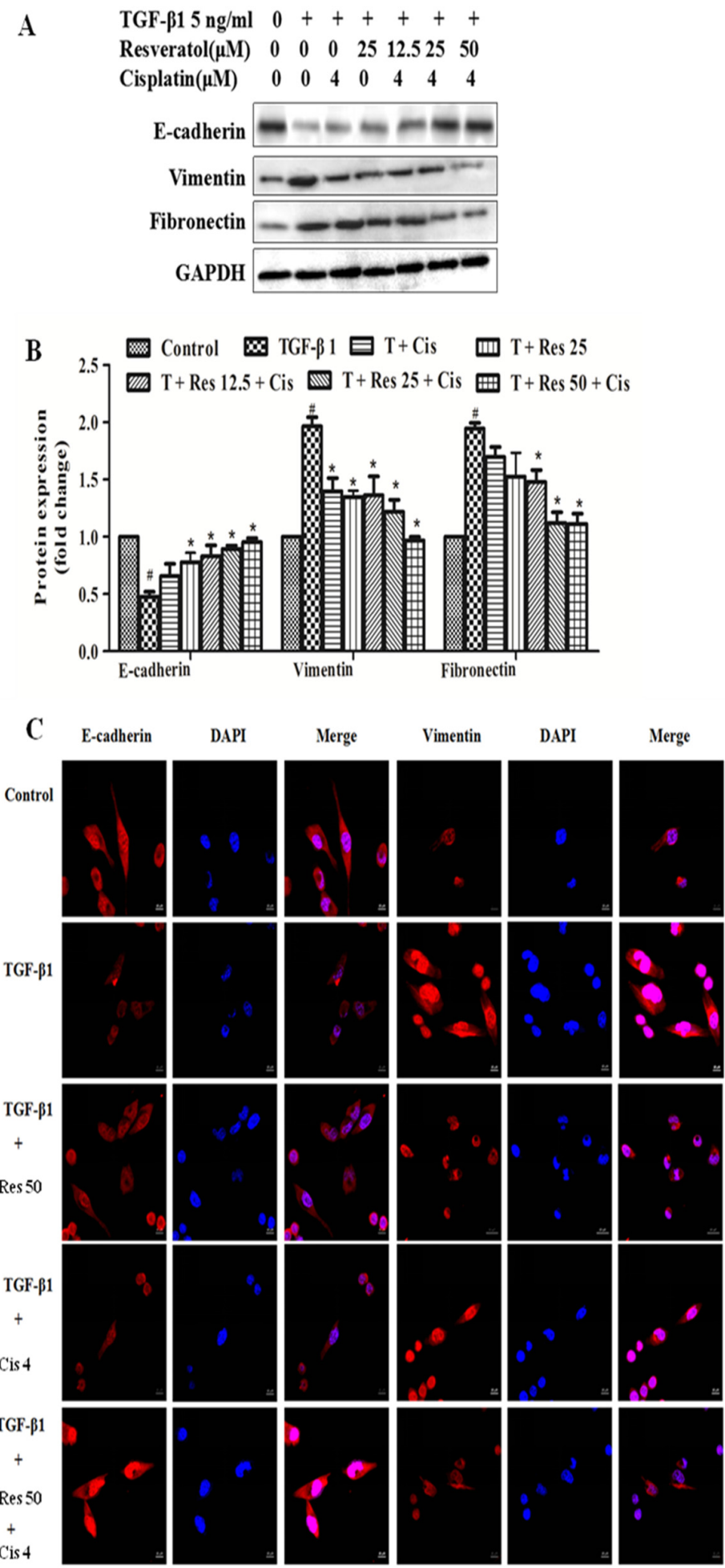

Figure 3. Regulatory effect of resveratrol combined with cisplatin on TGF- $\beta 1$-induced epithelial and mesenchymal molecular markers in MDA231 cells. (A) TGF- $\beta 1$-induced expressions of E-cadherin, vimentin and fibronectin treated by $5 \mathrm{ng} / \mathrm{mL}$ TGF- $\beta 1,12.5 \mu \mathrm{M}, 25 \mu \mathrm{M}, 50 \mu \mathrm{M}$ resveratrol, $4 \mu \mathrm{M}$ cisplatin or resveratrol + cisplatin + TGF- $\beta 1$ for $24 \mathrm{~h}$. (B) Protein expression fold change. The experiment was repeated three times, $\# p<0.01$, vs. control group; ${ }^{*} p<0.05$, vs. TGF- $\beta 1$-treated group. (C) TGF- $\beta 1$-induced expressions of E-cadherin and vimentin with immunofluorescence assay, treated by $50 \mu \mathrm{M}$ resveratrol and $4 \mu \mathrm{M}$ cisplatin for $24 \mathrm{~h}$. 
Red represents E-cadherin and vimentin, and blue represents DAPI. Photographed under a laser confocal microscope $(\times 625)$.

2.4. PI3K/AKT, Smad, NF- $\kappa B$, JNK, ERK Signal Pathways May Involve in TGF- $\beta 1$-Induced EMT by the Regulation of Resveratrol and Cisplatin in MDA231 Cells

In order to demonstrate the effect of resveratrol and cisplatin on the migration and invasion of MDA231 cells whether through EMT approach, $5 \mathrm{ng} / \mathrm{mL}$ TGF- $\beta 1$-induced EMT was treated by $25 \mu \mathrm{M}$ resveratrol, $4 \mu \mathrm{M}$ cisplatin, and within or without $10 \mu \mathrm{M}$ LY290042 (PI3K inhibitor), $10 \mu \mathrm{M}$ SB431542 (Smad inhibitor), $10 \mu \mathrm{M}$ PDTC (NF-kB inhibitor), $10 \mu \mathrm{M}$ SP600125 (JNK Inhibitor), $10 \mu \mathrm{M}$ PD98059 (ERK inhibitor) for $24 \mathrm{~h}$ and then the expression of epithelial and mesenchymal marker proteins were observed by western blot. As shown in Figure $4 \mathrm{~A}$, the expression of E-cadherin was decreased and the expression of vimentin and fibronectin were increased compared to control group $(p<0.01)$. When resveratrol and cisplatin were combined with LY290042 or SB431542 reversed the expression of these proteins induced by TGF- $\beta 1$. The results showed that the effects of resveratrol and cisplatin on the EMT induced by TGF- $\beta 1$ in MDA231 cells may be involved in the regulated of PI3K and Smad signaling pathways.

Furthermore, the resveratrol and cisplatin regulated the proteins expressions of EMTrelated pathways in MDA231 cells were demonstrated by western blot. As shown in Figure 4B, the expression of P-AKT, P-PI3K, Smad2, Smad3, P-JNK, and P-ERK were increased in cells induced by TGF- $\beta 1$. The combination of resveratrol $25 \mu \mathrm{M}$ with TGF- $\beta 1$ and cisplatin reversed the expression of these proteins and was superior to the treatment of cisplatin alone. The results indicate that the effects of resveratrol combined with cisplatin on the EMT induced by TGF- $\beta 1$ in MDA231 cells may be involved in the regulation of $\mathrm{PI} 3 \mathrm{~K} / \mathrm{AKT}$ and Smad, as well as related to the regulation of NF-KB, JNK, and ERK.

\subsection{Resveratrol Enhances Anti-Tumor and Reduces Side Effects of Cisplatin in MDA231 Xenografts}

In order to demonstrate the anti-tumor effect of resveratrol and cisplatin, MDA231 xenografts were prepared and the effects of resveratrol combined with cisplatin were assessed. As shown in Figure 5A, the effect of $50 \mathrm{mg} / \mathrm{kg}$ resveratrol combined with $5 \mathrm{mg} / \mathrm{kg}$ cisplatin was superior to cisplatin alone, which reduced significantly the tumor weight compared to model group $(p<0.01)$ and cisplatin-treated group $(p<0.05)$ in MDA231 xenografts from 3 to 8 weeks, while resveratrol alone was no effect. As shown in Figure 5B, the body weights of MDA231 xenografts were significantly increased by the combination treatments of resveratrol and cisplatin compared to cisplatin-treated group $(p<0.05)$, while the body weights were significantly decreased by cisplatin $(p<0.05)$. As shown in Figure 5C, serum BUN and Cr were decreased by the combination treatments of resveratrol and cisplatin compared to cisplatin-treated group $(p<0.05)$, while the serum BUN and $\mathrm{Cr}$ were significantly increased by cisplatin $(p<0.05)$. There were no significant changes of ALT and AST in each treatment group $(p>0.05)$. These results indicated that resveratrol can reduce body weight loss and kidney function impairment by cisplatin.

2.6. Resveratrol Combined with Cisplat Inhibits the Expression of P-AKT, P-PI3K, Smad2, Smad3, $P$-JNK, P-ERK, and NF- $\kappa B$ in Tumor Tissues of MDA231 Xenografts

In order to investigate the mechanisms of anti-tumor effect of resveratrol and cisplatin, the proteins expressions of tumor proliferation pathways in tumor tissues of MDA231 xenografts were demonstrated by western blot. As shown in Figure 6, the expressions of P-AKT, P-PI3K, Smad2, Smad3, P-JNK, P-ERK, and NF- $\mathrm{kB}$ were decreased by resveratrol and resveratrol combined with cisplatin compared to the model group, and the effects of the combination were better than in the resveratrol alone treatment. $(p<0.05)$, but there were no significant changes between cisplatin and model groups $(p>0.05)$. The results indicated that the regulations of P-AKT, P-PI3K, Smad2, Smad3, P-JNK, P-ERK, and NF- $\mathrm{KB}$ expressions may be involved in resveratrol enhances anti-tumor of cisplatin in MDA231 xenografts. 


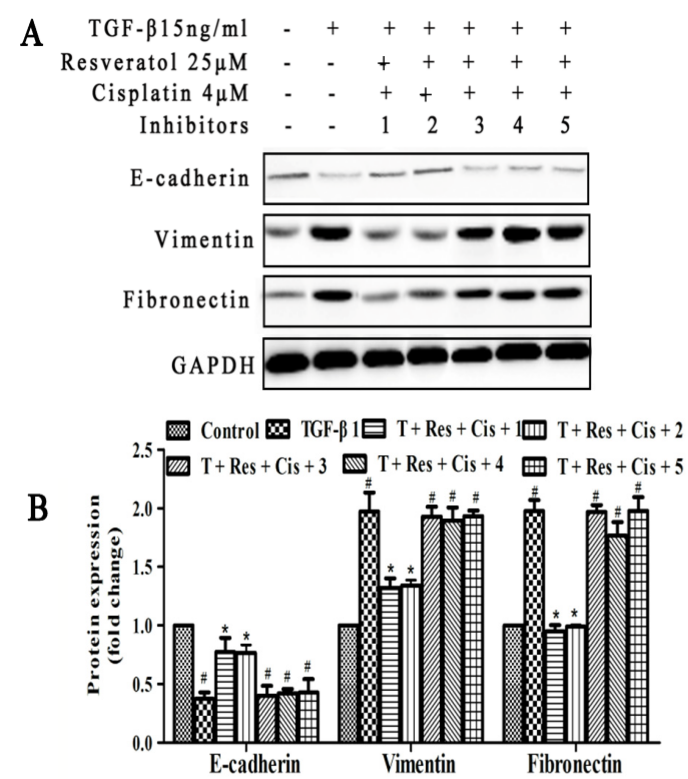

C
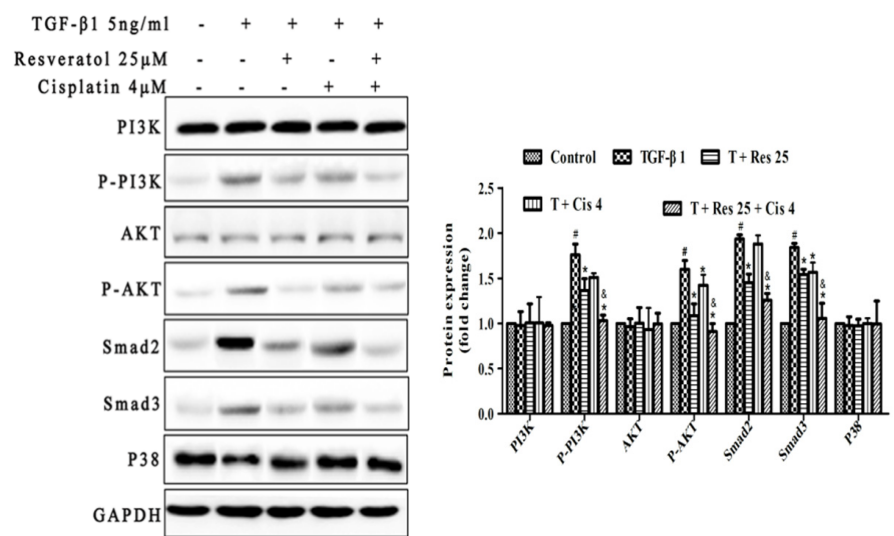

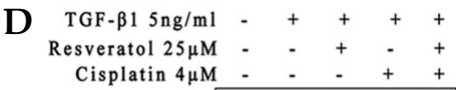
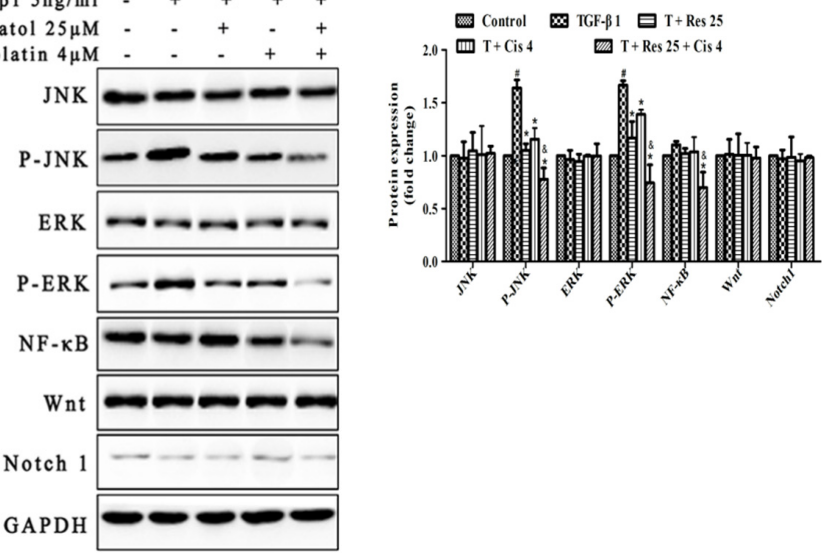

Figure 4. Effects of resveratrol and cisplatin on EMT-related pathway proteins induced by TGF- $\beta 1$ in MDA231 cells. (A) Expressions of epithelial and mesenchymal markers by the treatment of signaling pathway inhibitors; (C) Expressions of EMT-related pathway proteins (B,D) protein expression fold change. T, TGF- $\beta 15$ ng/mL; 1, LY290042; 2, SB431542; 3, SP600125; 4, PD98059; 5, PDTC. The experiment was repeated three times, \# $p<0.01$, vs. control group; ${ }^{*} p<0.05$, vs. TGF- $\beta 1$-treated group; \& $p<0.01$, compared to TGF- $\beta 1+$ cisplatin-treated group. 

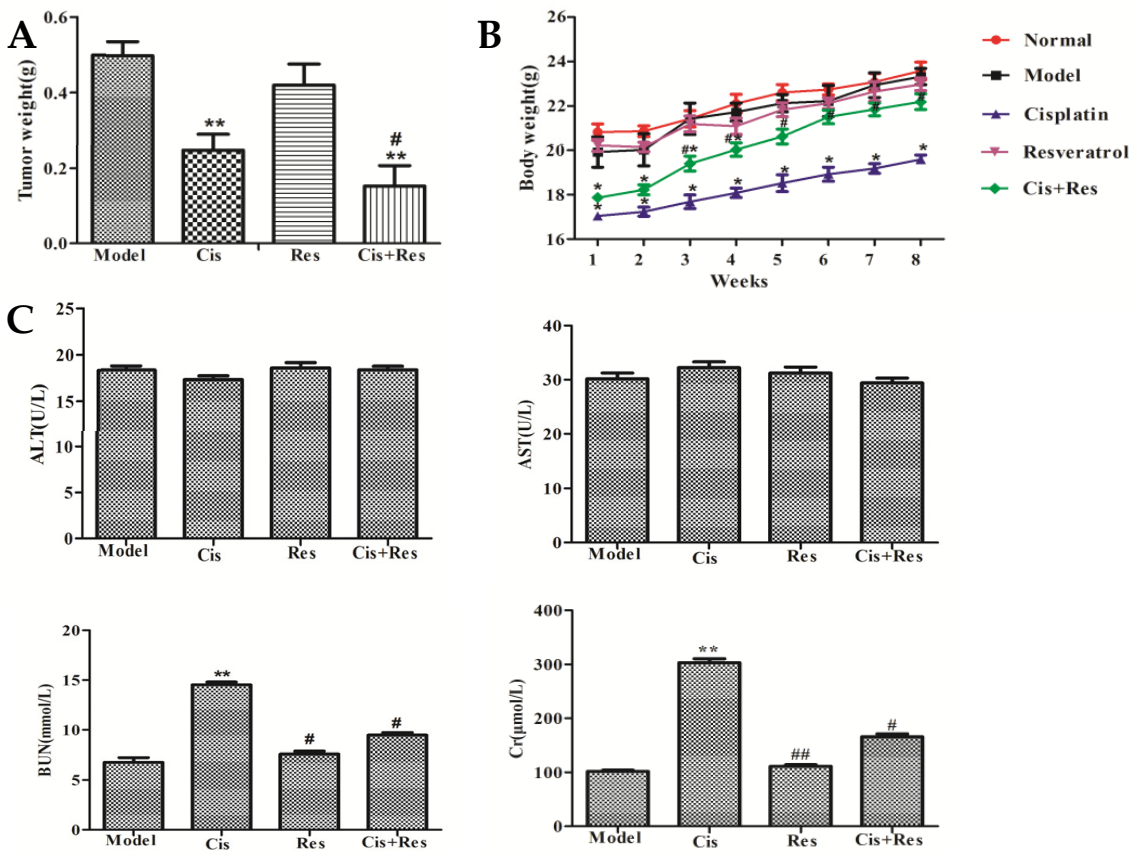

Figure 5. Anti-tumor effect of resveratrol and cisplatin without side effects in MDA231 xenografts. (A) Effects on tumor weight; (B) effects on body weight; (C) effects on serum ALT, AST, BUN, and Cr. ** $p<0.01,{ }^{*} p<0.05$ vs. model group; \#\# $p<0.01$, \# $p<0.05$, vs. cisplatin-treated group.
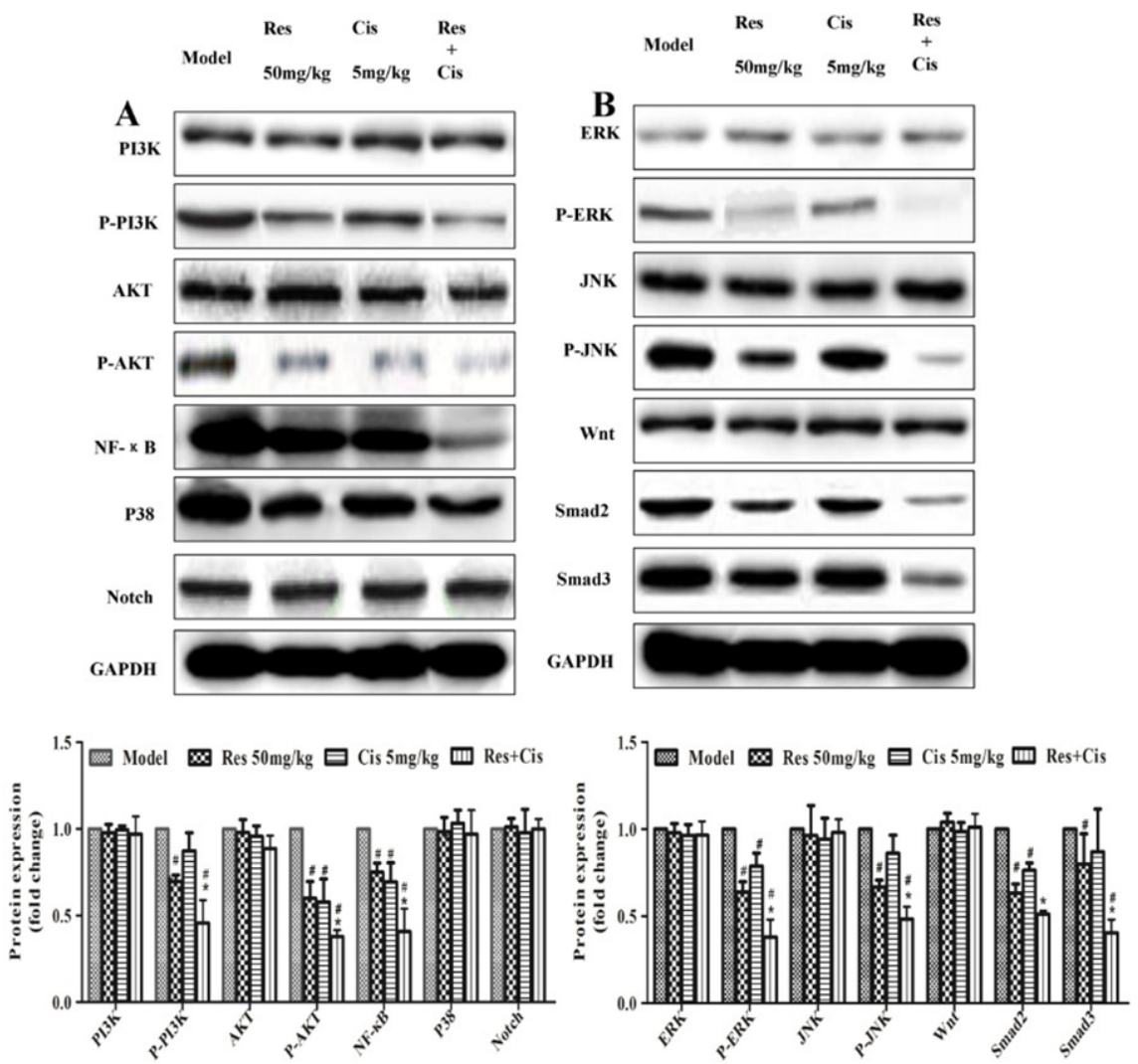

Figure 6. Effect of resveratrol and cisplatin on the expression of P-AKT, P-PI3K, Smad2, Smad3, P-JNK, P-ERK, and NF- $\kappa B$ in tumor tissues of MDA231 xenografts. (A) Fragments of each protein expressions by western blot analysis; (B) Fold changes of protein expressions. The experiment was repeated three times. ${ }^{*} p<0.05$ vs. model group; $\# p<0.05$, vs. cisplatin-treated group. 


\section{Materials and Methods}

\subsection{Reagents}

MDA-MB-231 cell was purchased from the Shanghai Cell Bank of the Chinese Academy of Sciences (Shanghai, China). DMEM were purchased from Gibico (Brooklyn, NY, USA); MTS purchased from Promega (Madison, WI, USA); Smad2 and Smad3 were purchased from Santa Cruz; Fibronectin, P-PI3K, PI3K, P38, P-AKT, AKT, E-cadherin, Vimentin, Notch1, Wnt and NF-kB were purchased from Cell Signaling Technology (Danvers, MA, USA); LY290042, SB431542, SP600125 and PD98059 PDTC were purchased from Selleck Chemical Company (Houston, TX, USA); SB203580, Cyclopamin, ETC-159 and DAPT were purchased from MedChemExpresss (Monmouth Junction, NJ, USA); HRP-labeled goat anti-rabbit and anti-mouse IgG were purchased from Beijing Boda Tektronix Biogene Technology Co., Ltd. (Beijing, China); Resveratrol (98\% content) was purchased from Shanghai Traditional Chinese Medicine Standardization Research Center (Shianghai, China). Cisplatin was purchased from Qilu Pharmaceutical (Hainan, China). IRDye ${ }^{\mathrm{TM}}$ Fluorescent Antibody was purchased from Li-Cor Bioscience (Lincoin, NE, USA); Ultrasensitive Chemiluminescence Detection Kit was purchased from Shanghai Ya Enzyme Co., Ltd. (Shanghai, China); Immunofluorescence staining blocking solution (Containing DAPI) was purchased from Shanghai Biyuntian Company (Shanghai, China).

\subsection{Cell Viability Assay}

MDA231 cells of $5 \times 10^{7} / \mathrm{L}$. were inoculated $200 \mu \mathrm{L}$ in each hole on 96-well culture plates. Resveratrol group with final concentration of $0,12.5,25$, and $50 \mu \mathrm{M}$, cisplatin group of $10,20,40,60,80,100 \mu \mathrm{M}, 14 \mu \mathrm{M}$ of cisplatin combined with resveratrol, $18 \mu \mathrm{M}$ of resveratrol combined with cisplatin were set up according to the experimental requirements, each concentration has four compound holes. After 24, 48, and $72 \mathrm{~h}$ of incubation, $20 \mathrm{~mL}$ MTS was added to each well. The absorbance value (OD value) was measured at $490 \mathrm{~nm}$. Calculate the combination indices (CIs) were analyzed using CompuSyn software (ComboSyn Inc., Paramus, NJ, USA). The CI value $<1$ is a synergistic effect, the CI value $=1$ is an additive effect, and the CI value $>1$ is an antagonistic effect.

\subsection{Invasion and Migration Assay}

In the invasion experiment, $30 \mu \mathrm{g}$ of Matrigel was applied to the upper layer of the chamber. Adjust the cell concentration to $1 \times 10^{5} / \mathrm{mL}$, add $200 \mu \mathrm{L}$ of cell suspension with or without drug to the upper layer of the 24-well Transwell chamber. Aspirate the culture solution after $24 \mathrm{~h}$, wipe off cells that have not invaded or migrated in the upper layer of the chamber by cotton swab. Rinse cells twice with PBS and fix with $4 \%$ paraformaldehyde for $15 \mathrm{~min}$, then dye with $0.1 \%$ crystal violet for half an hour. Finally, the photo is counted and take semi-quantitative experiment.

\subsection{Western Blot Assay}

Whole cell lysates or tissue homogenate were electrophoresed, incubated and assayed as described in previous literature [12], The primary antibodies including E-cadherin, vimentin, fibronectin, AKT, P-AKT, PI3K, P-PI3K, Smad2, Smad3, ERK, P-ERK, P38, JNK, P-JNK, NF-KB, Wnt, Notch1 (all of 1:1000 attenuation), and secondary antibody GAPDH (1:5000 attenuation) were used. The assays were repeated at least three times.

\subsection{Immunofluorescence Assay}

MDA-MB-231 cells were adjusted to a cell density of $2.5 \times 10^{5}$ cells $/ \mathrm{mL}$, and the cells were cultured on confocal dishes treated with TGF- $\beta 1(5 \mathrm{ng} / \mathrm{mL}), \mathrm{TGF}-\beta 1+$ resveratrol $(50 \mu \mathrm{M})$, TGF- $\beta 1+$ cisplatin $(40 \mu \mathrm{M})$ or TGF- $\beta 1+$ resveratrol + cisplatin for $24 \mathrm{~h}$ fixed with $4 \%$ paraformaldehyde for $30 \mathrm{~min}$, and then stabilized in $0.5 \%$ Triton $\mathrm{X}-100$ for $20 \mathrm{~min}$. After three PBS washes and blocking with Quick Block ${ }^{\mathrm{TM}}$ Blocking Buffer for Immunol Staining for $15 \mathrm{~min}$, the cells were incubated with E-cadherin (1:200) and anti-Vimentin (1:500) antibodies overnight at $4{ }^{\circ} \mathrm{C}$. After washing, the cells were blocked from light, 
incubated with an anti-rabbit antibody for $60 \mathrm{~min}$ and counterstained with DAPI. The cells were observed and photographed with a confocal fluorescence microscope (LSM880, Zeiss, Jena, Germany).

\subsection{Preparation, Administration, and Treatment of MDA231 Xenografts}

Seven-week-old female BALB/c mice (18-23 g) were fed at the Laboratory Animal Center at Shanghai University of Traditional Chinese Medicine. The preparation and administration of MDA231 Xenografts was as described in previous literature [12]. The mice were injected once every two days (days for Cisplatin) with $100 \mathrm{mg} / \mathrm{kg}$ resveratrol (resveratrol group, $n=10$ ), $50 \mathrm{mg} / \mathrm{kg}$ cisplatin (Cisplatin group, $n=10$ ) $100 \mathrm{mg} / \mathrm{kg}$ resveratrol $+50 \mathrm{mg} / \mathrm{kg}$ cisplatin (Cisplatin group, $n=10$ ) or PBS for MDA231 Xenografts (model group, $n=5$ ) and PBS for BALB/c (normal group, $n=5$ ) through the peritoneal cavity. Body of the mice were measured once per week. After 8 weeks of tumor cell inoculation, the mice were sacrificed, and the tumors were removed and weighed.

\subsection{Liver and Kidney Function Tests}

When the mice were sacrificed, $1 \mathrm{~mL}$ blood was collected from the eyes and then quickly centrifuged for $10 \mathrm{~min}$ at $3000 \mathrm{rpm}$ to obtain the serum. The levels of serum ALT, AST, Cr, and BUN were detected according to the manufacturer's colorimeter testing kits (Jiancheng Bioengineering Institute, Nanjing, China).

\subsection{Statistical Analysis}

The experimental data were expressed as mean \pm standard deviation $(x \pm S D)$. The comparison of the two means was performed by $t$-test, and the comparison of multiple means was analyzed by variance. The difference was statistically significant at $p<0.05$. Statistical analysis was performed by SPSS 19.0 software.

\subsection{Ethics Approval and Consent to Participate}

All animal procedures were conducted in accordance with the guidelines of the National Institutes of Health and were approved by the Ethical Committee of the Shanghai University of Traditional Chinese Medicine (approval ID PZSHUTCM18-101804).

\section{Discussion}

TNBC refers to breast cancer that does not express the estrogen receptor (ER), progesterone receptor (PR), and HER2/neu genes [28]. Although TNBC is sensitive to chemotherapy, such as the platinum can significantly improve the prognosis of TNBC, but the serious side effects of platinum drugs are also a non-negligible fact in clinical applications [29]. Therefore, it is particularly necessary to find new drugs or synergistic combination strategies that are more effective against TNBC and can alleviate the side effects of chemotherapy drugs.

Chinese herbal medicines (one of natural medicines) have long been used in cancer therapy as such synergistic combinations for enhancing efficacy, reducing side effects, immune modulation, as well as abrogating drug resistance of chemotherapy [30]. Therein, resveratrol-based combinatorial strategies for cancer management has also been increasingly studied, that showed resveratrol sensitizes tamoxifen in antiestrogen-resistant breast cancer cells [11] and TNF- $\beta$-induced survival of 5-FU-treated colorectal cancer cells [31], increases arsenic trioxide-induced apoptosis in chronic myeloid leukemia cells [32] and lung cancer cells [33], decreases cytotoxicity of doxorubicin in breast cancer cells [15], and combined with piceatannol upregulates PD-L1 expression in breast and colorectal cancer cells [34]. However, the effects of resveratrol combined with cisplatin on breast cancer is still unclear.

In this study, we demonstrated the effects of resveratrol combined with cisplatin using a TNBC models, MDA231 cells in vivo and in vitro. Our results showed that, in vitro, resveratrol combined with cisplatin inhibits synergistically cell viability, and inhibits TGF $\beta 1$-induced cell migration and invasion (Figures 1 and 2); in vivo, resveratrol enhanced 
tumor growth inhibition and reduced body weight loss and kidney function impairment by cisplatin in MDA231 xenografts (Figure 5). These results indicated that resveratrol may sensitize the inhibitive effects of cisplatin on cell viability, migration and invasion, and tumor growth in MDA231 models in vivo and in vitro.

EMT is a process in which epithelial cells lose cell polarity and intercellular adhesion, gain migration, and invasiveness and become interstitial cells. Epithelial cells express high levels of E-cadherin, while mesenchymal cells express $\mathrm{N}$-cadherin, fibronectin, and vimentin. Thus, EMT causes morphological and phenotypic changes in cells [35]. EMT plays an important role in the process of TNBC. It has been reported that resveratrol can reverse EMT [12], and sensitizes tamoxifen in antiestrogen-resistant with EMT in breast cancer cells [11]. In this study, we found that resveratrol sensitizes the effects of cisplatin inhibits TGF $\beta 1$-induced MDA231 cell migration and invasion by reducing the expression of vimentin, fibronectin, and increased the expression of E-cadherin, which is reversing EMT, while there is no obvious effect in those of cisplatin alone (Figure 3). It indicated that resveratrol gives cisplatin an efficacy of reversing EMT.

There are multiple dysregulated signaling pathways such as Wnt/ $\beta$-catenin, Notch, NF- $\mathrm{B}$, PI3K/Akt, Smad, MAPK (including p38, JNK and ERK) and Hedgehog in TNBC, and these signaling pathways involved in the regulation of cell growth, proliferation, migration, EMT and metastasis, and activation of apoptosis, and affected by natural compounds such as resveratrol or its combination with classical chemotherapeutic agents in TNBC [36]. In order to clarify which signaling pathway involved in the regulation of EMT treated by resveratrol and cisplatin combination in MDA231 cells, we screened the TGF- $\beta 1$-induced EMT-related signaling pathways, using inhibitors including LY290042 for PI3K, SB431542 for Smad, PDTC for NF-кB, SP600125 for JNK, and PD98059 for ERK. Our results showed that, PI3K and Smad signaling pathways may be involved in the regulated of EMT induced by TGF- $\beta 1$ in MDA231 cells (Figure 4A). Moreover, further experiments showed the combination of resveratrol and cisplatin downregulated the expression of P-JNK, and P-ERK, which increased in MDA231 cells induced by TGF- $\beta 1$ (Figure 4B), indicated the effects on EMT were involved in the regulation of PI3K/AKT and Smad signal pathways, as well as regulated NF- $\mathrm{KB}, \mathrm{JNK}$, and ERK expressions.

Moreover, previous studies have reported that resveratrol inhibits tumor growth by inducing apoptosis in MDA231 xenograft and HER-2/neu transgenic mice models [37,38]. The inhibitive tumor effects of resveratrol combined with quercetin and catechin by the regulation of cell cycle progression in MDA231 xenograft has also been reported [39]. However, the tumor-inhibited mechanisms of resveratrol combined with cisplatin on breast cancer is still unclear. In this study, we found that resveratrol enhanced the inhibitive effects of cisplatin on P-AKT, P-PI3K, Smad2, Smad3, P-JNK, P-ERK, and NF-кB expressions in MDA231 xenografts (Figure 6) indicated that the regulations of P-AKT, P-PI3K, Smad2, Smad3, P-JNK, P-ERK, and NF- $\mathrm{BB}$ expressions may be involved in resveratrol enhances antitumor of cisplatin in MDA231 xenografts. In addition, because resveratrol can inhibit tumor metastasis [12] and multiple signal pathway involved in metastasis of breast cancer [8,36], further study will investigate the effects and mechanisms of Resveratrol combined with cisplatin on the tumor metastasis.

\section{Conclusions}

In summary, resveratrol combined with cisplatin produced a synergistic effect on the inhibition of breast cancer cell viability, inhibits breast cancer MDA231 cell migration and invasion through EMT regulated by PI3K/AKT, Smad, NF- $\mathrm{B}$, JNK, and ERK. Moreover, resveratrol enhanced anti-tumor effect and reduced side of cisplatin in MDA231 xenografts and the effective mechanism may be involved in the regulations of PI3K/AKT, JNK, ERK, and NF- $\mathrm{kB}$ expressions. 
Author Contributions: Conceptualization, S.-B.S.; Data collocation and analysis, Y.S., M.-D.Y., W.-J.Z., X.-Z.X., Q.-M.Z. and Y.-Y.L.; Writing-original draft, M.-D.Y.; Writing-review and editing, Y.S. and S.-B.S. All authors have read and agreed to the published version of the manuscript.

Funding: This research was supported by the Key Program of National Natural Science Fund of China (no. 81330084).

Institutional Review Board Statement: The Institutional Review Board Statement and approval number is PZSHUTCM190308007.

Informed Consent Statement: Informed consent was obtained from all subjects involved in the study.

Data Availability Statement: The data presented in this study are available with the authors.

Conflicts of Interest: The author(s) confirm that this article content has no conflict of interest.

Sample Availability: The samples are available from the authors.

\section{References}

1. Li, T.; Mello-Thoms, C.; Brennan, P.C. Descriptive epidemiology of breast cancer in China: Incidence, mortality, survival and prevalence. Breast Cancer Res. Treat. 2016, 159, 395-406. [CrossRef] [PubMed]

2. Momenimovahed, Z.; Salehiniya, H. Epidemiological characteristics of and risk factors for breast cancer in the world. Breast Cancer 2019, 11, 151-164. [CrossRef] [PubMed]

3. Kwa, M.J.; Adams, S. Checkpoint inhibitors in triple-negative breast cancer (TNBC): Where to go from here. Cancer 2018, 124, 2086-2103. [CrossRef]

4. $\quad$ Kennecke, H.; Yerushalmi, R.; Woods, R.; Cheang, M.C.U.; Voduc, D.; Speers, C.H.; Nielsen, T.O.; Gelmon, K. Metastatic behavior of breast cancer subtypes. J. Clin. Oncol. 2010, 28, 3271-3277. [CrossRef] [PubMed]

5. Jiao, Q.; Wu, A.; Shao, G.; Peng, H.; Wang, M.; Ji, S.; Liu, P.; Zhang, J. The latest progress in research on triple negative breast cancer (TNBC): Risk factors, possible therapeutic targets and prognostic markers. J. Thorac. Dis. 2014, 6, 1329-1335.

6. Park, J.H.; Ahn, J.H.; Kim, S.B. How shall we treat early triple-negative breast cancer (TNBC): From the current standard to upcoming immuno-molecular strategies. ESMO Open 2018, 3 (Suppl. 1), e000357. [CrossRef] [PubMed]

7. Dent, R.; Trudeau, M.; Pritchard, K.I. Triple-negative breast cancer: Clinical features and patterns of recurrence. Clin. Cancer Res. 2007, 13, 4429-4434. [CrossRef]

8. Ko, J.H.; Sethi, G.; Um, J.Y.; Shanmugam, M.K.; Arfuso, F.; Kumar, A.P.; Bishayee, A.; Ahn, K.S. The role of resveratrol in cancaer therepy. Int. J. Mol. Sci. 2017, 18, 2589. [CrossRef] [PubMed]

9. Fu, Y.; Chang, H.; Peng, X.; Bai, Q.; Yi, L.; Zhou, Y.; Zhu, J.; Mi, M. Resveratrol inhibits breast cancer stem-like cells and induces autophagy via suppressing Wnt/-catenin signaling pathway. PLoS ONE 2014, 9, e102535. [CrossRef] [PubMed]

10. Sprouse, A.; Herbert, B. Resveratrol augments paclitaxel treatment in MDA-MB-231 and paclitaxel-resistant MDA-MB-231 breast cancer cells. Anticancer Res. 2014, 3, 5363-5374.

11. Shi, X.P.; Miao, S.; Wu, Y.; Zhang, W.; Zhang, X.F.; Ma, H.Z.; Xin, H.L.; Feng, J.; Wen, A.D.; Li, Y. Resveratrol sensitizes tamoxifen in antiestrogen-resistant breast cancer cells with epithelial-mesenchymal transition features. Int. J. Mol. Sci. 2013, 14, 15655-15668. [CrossRef]

12. Sun, Y.; Zhou, Q.-M.; Lu, Y.-Y.; Zhang, H.; Chen, Q.-L.; Zhao, M.; Su, S.-B. Resveratrol inhibits the migration and metastasis of MDA-MB-231 human breast Cancer by reversing TGF- 31 -induced epithelial-mesenchymal transition. Molecules 2019, $24,1131$. [CrossRef]

13. Chatterjee, A.; Ronghe, A.; Singh, B.; Bhat, N.K.; Chen, J.; Bhat, H.K. Natural antioxidants exhibit chemopreventive characteristics through the regulation of CNC b-Zip transcription factors in estrogen-induced breast carcinogenesis. J. Biochem. Mol. Toxicol. 2014, 28, 529-538. [CrossRef]

14. Mirza, S.; Sharma, G.; Parshad, R.; Gupta, S.D.; Pandya, P.; Ralhan, R. Expression of DNA methyl transferases in breast cancer patients and to analyze the effect of natural compounds on DNA methyltransferases and associated proteins. J. Breast Cancer 2013, 16, 23-31. [CrossRef]

15. Osman, A.M.; Bayoumi, H.M.; AI-Harthi, S.E.; Damanhouri, Z.A.; Elshal, M.F. Modulation of doxorubicin cytotoxicity by resveratrol in a human breast cancer cell line. Cancer Cell Int. 2012, 12, 47. [CrossRef]

16. Vinod, B.S.; Nair, H.H.; Vijayakurup, V.; Damanhouri, Z.A.; Elshal, M.F. Resveratrol chemosensitizes her-2-overexpressing breast cancer cells to docetaxel chemoresistance by inhibiting docetaxel-mediated activation of her-2-Akt axis. Cell Death Discov. 2015, 1, 15061. [CrossRef]

17. Malaguarnera, L. influence of resveratrol on the immune response. Nutrients 2019, 11, 946. [CrossRef] [PubMed] 
18. Han, X.J.; Yang, Z.J.; Jiang, L.P.; Wei, Y.; Liao, M.; Qian, Y.; Li, Y.; Huang, X.; Wang, J.; Xin, H.; et al. Mitochondrial dynamics regulates hypoxia-induced migration and antineoplastic activity of cisplatin in breast cancer cells. Int. J. Oncol. 2015, 46, 691-700. [CrossRef] [PubMed]

19. Pegram, M.D.; Lipton, A.; Hayes, D.F.; Weber, B.L.; Baselga, J.M.; Tripathy, D.; Baly, D.; Baughman, S.A.; Twaddell, T.; Glaspy, J.A.; et al. Phase II study of receptor-enhanced chemosensitivity using recombinant humanized anti-p185HER2/neu monoclonal antibody plus cisplatin in patients with HER2/neu-overexpressing metastatic breast cancer refractory to chemotherapy treatment. J. Clin. Oncol. 1998, 16, 2659-2671. [CrossRef] [PubMed]

20. Wiggans, R.G.; Woolley, P.V.; Smythe, T.; Hoth, D.; Macdonald, J.S.; Green, L.; Schein, P.S. Phase-II trial of tamoxifen in advanced breat cancer. Cancer Chemother. Pharmacol. 1979, 3, 45-48. [CrossRef] [PubMed]

21. Liang, F.; Zhang, S.; Xue, H.; Chen, Q. Risk of second primary cancers in cancer patients treated with cisplatin: A systematic review and meta-analysis of randomized studies. BMC Cancer 2017, 17, 1-12. [CrossRef]

22. Oh, G.S.; Kim, H.J.; Shen, A.; Lee, S.B.; Khadka, D.; Pandit, A.; So, H.S. Cisplatin-induced Kidney Dysfunction and Perspectives on Improving Treatment Strategies. Electrolyte Blood Press. 2014, 12, 55-65. [CrossRef] [PubMed]

23. Sorenson, J.R.; Wangila, G.W. Co-treatment with copper compounds dramatically decreases toxicities observed with cisplatin cancer therapy and the anticancer efficacy of some copper chelates supports the conclusion that copper chelate therapy may be markedly more effective and less toxic than cisplatin therapy. Curr. Med. Chem. 2007, 14, 1499-1503. [PubMed]

24. Shahid, F.; Farooqui, Z.; Khan, F. Cisplatin-induced gastrointestinal toxicity: An update on possible mechanisms and on available gastroprotective strategies. Eur. J. Pharmacol. 2018, 827, 49-57. [CrossRef]

25. Donertas, B.; Unel, C.C.; Erol, K. Cannabinoids and agmatine as potential therapeutic alternatives for cisplatin-induced peripheral neuropathy. J. Exp. Pharmacol. 2018, 10, 19-28. [CrossRef]

26. Trendowski, M.R.; El Charif, O.; Dinh, P.C., Jr.; Travis, L.B.; Dolan, M.E. Genetic and modifiable risk factors contributing to cisplatin-induced toxicities. Clin. Cancer Res. 2019, 25, 1147-1155. [CrossRef]

27. Fiteni, F.; Nguyen, T.; Vernerey, D.; Paillard, M.; Kim, S.; Demarchi, M.; Fein, F.; Borg, C.; Bonnetain, F.; Pivot, X. Cisplatin/gemcitabine or oxaliplatin/gemcitabine in the treatment of advanced biliary tract cancer: A systematic review. Cancer Med. 2014, 3, 1502-1511. [CrossRef]

28. Foulkes, W.D.; Smith, I.E.; Reis-Filho, J.S. Triple-negative breast cancer. N. Engl. J. Med. 2010, 363, 1938-1948. [CrossRef] [PubMed]

29. Jiang, T.; Shi, W.; Wali, V.B.; Pongor, L.S.; Li, C.; Lau, R.; Győrffy, B.; Lifton, R.P.; Symmans, W.F.; Pusztai, L.; et al. Predictors of chemosensitivity in triple negative breast cancer: An integrated genomic analysis. PLoS Med. 2016, 13, e1002193. [CrossRef]

30. Hu, X.-Q.; Sun, Y.; Lau, E.; Zhao, M.; Su, S.-B. Advances in synergistic combinations of chinese herbal medicine for the treatment of cancer. Curr. Cancer Drug Targets 2016, 16, 346-356. [CrossRef]

31. Buhrmann, C.; Yazdi, M.; Popper, B.; Shayan, P.; Goel, A.; Aggarwal, B.B.; Shakibaei, M. Resveratrol Chemosensitizes TNF- $\beta$ Induced Survival of 5-FU-Treated Colorectal Cancer Cells. Nutrients 2018, 10, 888. [CrossRef]

32. Wu, E.J.; Goussetis, D.J.; Beauchamp, E.; Kosciuczuk, E.M.; Altman, J.K.; Eklund, E.A.; Platanias, L.C. Resveratrol enhances the suppressive effects of arsenic trioxide on primitive leukemic progenitors. Cancer Biol. Ther. 2014, 15, 473-478. [CrossRef] [PubMed]

33. Gu, S.; Chen, C.; Jiang, X.; Zhang, Z. Resveratrol synergistically triggers apoptotic cell death with arsenic trioxide via oxidative stress in human lung adenocarcinoma A549 cells. Biol. Trace. Elem. Res. 2015, 163, 112-123. [CrossRef] [PubMed]

34. Lucas, J.; Hsieh, T.-C.; Halicka, H.D.; Darzynkiewicz, Z.; Wu, J.M. Upregulation of PD-L1 expression by resveratrol and piceatannol in breast and colorectal cancercells occurs via HDAC3/p300-mediated NF-kB signaling. Int. J. Oncol. 2018, 53, 1469-1480. [PubMed]

35. Samy, L.; Jian, X.; Rik, D. Molecular mechanisms of epithelial-mesenchymal transition. Nat. Rev. Mol. Cell Biol. 2014, 15, 178-196.

36. Varghese, E.; Samuel, S.M.; Abotaleb, M.; Cheema, S.; Mamtani, R.; Büsselberg, D. The "Yin and Yang" of natural compounds in anticancer therapy of triple-negative breast cancers. Cancers 2018, 10, 346. [CrossRef] [PubMed]

37. Garvin, S.; Ollinger, K.; Dabrosin, C. Resveratrol induces apoptosis and inhibits angiogenesis in human breast cancer xenografts in vivo. Cancer Lett. 2006, 231, 113-122. [CrossRef]

38. Provinciali, M.; Re, F.; Donnini, A.; Orlando, F.; Bartozzi, B.; Di Stasio, G.; Smorlesi, A. Effect of resveratrol on the development of spontaneous mammary tumors in HER-2/neu transgenic mice. Int. J. Cancer 2005, 115, 36-45. [CrossRef]

39. Schlachterman, A.; Valle, F.; Wall, K.M.; Azios, N.G.; Castillo, L.; Morell, L.; Washington, A.V.; Cubano, L.A.; Dharmawardhane, S.F. Combined resveratrol, quercetin, and catechin treatment reduces breast tumor growth in a nude mouse model. Transl. Oncol. 2008, 1, 19-27. [CrossRef] 